\title{
Post-meal affective states
}

\author{
Margarita Sala
}

Received: 9 January 2015/Accepted: 11 January 2015/Published online: 29 January 2015

(C) Springer International Publishing Switzerland 2015

Anderson and collaborators [1] investigated how meal consumption impacts the affective states of individuals with eating disorders. They administered the State-Trait Anxiety Inventory (STAI-S), the Positive and Negative Affect Schedule (PANAS), and the Profile of Mood States (POMS) to assess affective states pre- and post-meal. Individuals with binge eating disorder (BED) experienced decreases in negative affect after meal consumption, whereas individuals with anorexia nervosa (AN) and bulimia nervosa $(\mathrm{BN})$ experienced no changes in affect after meal consumption.

However, Anderson and collaborators' [1] findings may have been biased due to the affective measures that they used. The use of the PANAS, POMS, and STAI for measuring affect has been criticized on the basis that it may not capture all relevant affective states [2], and it may be useful to examine the limitations of relying solely on these instruments to measure post-meal affective states in the eating disorders field. Some states which are not captured by the PANAS may be relevant to the post-meal experiences of individuals with eating disorders (e.g. satisfaction, sadness). The POMS captures only six distinct mood states, and there are some mood states not captured by the POMS that may be influenced by the consumption of a meal (e.g. cheerfulness, regret). The STAI would not capture positively valenced increases in activation or negatively valenced decreases in activation post-meal consumption [3]. Although these measures are well validated and widely

M. Sala $(\bowtie)$

Department of Psychology, Southern Methodist University, 6116 N. Central Expressway, Suite 1300, Dallas, TX 75206, USA

e-mail: msala@smu.edu used, it is unclear whether these are the best measures to use in the context of eating disorders.

Thus, it is possible that meal consumption influences affective states not tapped by the POMS, PANAS, or STAI. Additional research evaluating the association of affective states not covered by these measures with eating pathology is needed. Future research should also examine basic affect [2]. It is unclear which post-meal affective states would be most clearly relevant to individuals with eating disorders, and focusing on only some selected affective states could result in failing to measure a significant affective state [2].

Conflict of interest The author declares that there are no conflicts of interest.

Ethical approval This article does not contain any studies with human participants or animals performed by any of the authors.

\section{References}

1. Anderson LM, Crow SJ, Peterson CB (2014) The impact of meal consumption on emotion among individuals with eating disorders. Eat Weight Disord 19(3):347-354. doi:10.1007/s40519-0130084-1

2. Ekkekakis P (2013) The measurement of affect, mood, and emotion: a guide for health-behavioral research. Cambridge University Press, Cambridge

3. Ekkekakis P, Petruzzello SJ (2002) Analysis of the affect measurement conundrum in exercise psychology: IV. A conceptual case for the affect circumplex. Psychol Sport Exerc 3(1):35-63. doi:10.1016/S1469-0292(01)00028-0 